\title{
ULTRASONIC WAVE PROPAGATION VELOCITY IN NONCONDUCTING LIQUIDS IN AN EXTERNAL DC ELECTRIC FIELD
}

\author{
M. Labowski, A. Skumiel and T. Hornowski \\ Institute of Acoustics, A. Mickiewicz University \\ Jana Matejki 48/49, 60-769 Poznań, Poland
}

(Received December 28, 1990; in revised form May 22, 1991)

\begin{abstract}
A theorelical analysis of variations in the propagation velocity of ultrasonic waves in liquid dieleçtrics in an external DC electric field was performed. Measurements of these variations in transformer oil in dependence on the field strength and temperature were made applying the phase method. To express the experimental results in graphical form numerical procedure of interpolation in Lagrange polynomials was used. The experimentally obtained variations in the velocity are compared with those predicted theoretically by the formulae derived.
\end{abstract}

PACS numbers: $43.35 .+d$

1. Effect of an external DC electric field on the ultrasonic propagation velocity in non-conducting liquids

From the point of view of thermodynamics, in order to measure the ultrasonic propagation velocity, the system has to be led to equilibrium; the equations of electrostatics are then fulfilled [1]. With regard to the IIelmholtz formula [2, 3] for the square of the propagation velocity of an acoustic wave:

$$
c^{2}=\frac{\kappa V^{2}}{M}\left(\frac{\partial^{2} F}{\partial V^{2}}\right)_{T},
$$

where $F$ is the molar free energy and $V$ - the molar volume of the liquid, we have to find the form of the free energy $F$ of the system as a function of state. The general formula is well known from thermodynamics, where the total free energy of 
the system is made to comprise an energy term dependent on the external electric field.

For reversible processes taking into account the conditions of equilibrium as well as the circumstance that the local electric field strength $\boldsymbol{F}$ is identical throughout the whole volume of the measuring vessel, we have [1]:

$$
F=U-S T+p V-\frac{V}{4 \pi} \varepsilon F^{2},
$$

where $U$ is the internal energy of the system, $V$ - its volume, $\boldsymbol{F}$ - the local field in the dielectric, $\varepsilon=\varepsilon_{r}, \varepsilon_{0}$ - the absolute electric permittivity of the dielectric, $p$ - the external pressure, $S$ - the entropy, and $T$ - temperature. On the basis of the Eq. (2) we arrive at the following expression for the derivative of the function of state

$$
\frac{\mathrm{d} F}{\mathrm{~d} V}=-p-\varepsilon_{0} \varepsilon_{r} F^{2}
$$

- On insertion of this derivative into the IIelmholtz formula (1) we get:

$$
c_{E}^{2}=\frac{\kappa}{\rho \beta_{\text {is }}}+\frac{\kappa \varepsilon_{0}}{\rho^{2}} \frac{\partial\left(\varepsilon_{r} F^{2}\right)}{\partial\left(\frac{1}{\rho}\right)},
$$

where $\kappa=c_{p} / c_{v}, M$ - the molar mass, $c_{E}$ - the ultrasonic propagation velocily in the presence of the external electric field, $\beta_{\text {is }}$ - isothermal compressibility. Equation (4) can also be written in the following form:

$$
c_{E}^{2}=c_{0}^{2}-\frac{\kappa \varepsilon_{0}}{2} \frac{\partial\left(\varepsilon_{r} F^{2}\right)}{\cdot \partial \rho}
$$

where $c_{0}^{2}$ is the square of the ultrasonic propagation velocity in the absence of an external electric field.

Let us now consider the density-derivative in Eq. (5) separately. On introducing the Lorentz field given by [4]:

$$
\boldsymbol{F}=\frac{\varepsilon_{r}+2}{3} E
$$

we get

$$
\frac{\partial\left(\varepsilon_{r} F^{2}\right)}{\partial \rho}=\frac{\left(\varepsilon_{r}+2\right)\left(3 \varepsilon_{r}+2\right)}{9}\left(\frac{\partial \varepsilon_{r}}{\partial \rho}\right)_{T} E^{2}+\frac{\varepsilon_{r}\left(\varepsilon_{r}+2\right)}{9}\left(\frac{\partial E^{2}}{\partial \rho}\right)_{T} .
$$

With the general Clausius-Mossotti formula, for $\mu=0$ and $\chi=\varepsilon_{r}-1$ we get:

$$
\frac{\varepsilon_{r}-1}{\varepsilon_{r}+2} \frac{M}{\rho}=\frac{N_{A}}{3 \varepsilon_{0}}\left(\alpha+\frac{\mu_{0}^{2}}{3 k T}\right)=\frac{N_{A} \alpha}{3 \varepsilon_{0}} .
$$

Transforming (7) to the form $\varepsilon_{r}=\varepsilon_{r}(\rho)$ and inserting $A$ for $N_{A} \propto / 3 \varepsilon_{0} M$ we obtain:

$$
\varepsilon_{r}=\frac{2 A \rho+1}{1-A \rho} \text {. }
$$


On the other hand, the derivative $\partial \varepsilon_{r} / \partial \rho$ is a quantity characteristic for the pressure due to electrostriction in a liquid dielectric [5]:

$$
p=\frac{\varepsilon_{0} E^{2}}{2}\left(\frac{\partial \varepsilon_{r}}{\partial \rho}\right)_{T} \rho
$$

Calculating the density-derivative of pressure we have:

$$
\left(\frac{\partial p}{\partial \rho}\right)_{T}=\frac{\varepsilon_{0} \rho}{2}\left(\frac{\partial \varepsilon_{r}}{\partial \rho}\right)_{T}\left(\frac{\partial E^{2}}{\partial \rho}\right)_{T}+E^{2} \rho \frac{\varepsilon_{0}}{2}\left(\frac{\partial^{2} \varepsilon_{r}}{\partial \rho^{2}}\right)_{T}+E^{2} \frac{\varepsilon_{0}}{2}\left(\frac{\partial \varepsilon_{r}}{\partial \rho}\right)_{T} .
$$

Since $(\mathrm{d} p / \mathrm{d} \rho)_{T}=(1 / \rho) \beta_{\mathrm{is}}$, where $\beta_{\mathrm{is}}$ is the isothermal compressibility of the liquid medium, we have:

$$
\left(\frac{d E^{2}}{\mathrm{~d} \rho}\right)_{T}=\left[\frac{2}{\left(\partial \varepsilon_{r} / \partial \rho\right)_{T} \rho^{2} \varepsilon_{0} \beta_{\mathrm{is}}}-E^{2} \frac{\left(\partial^{2} \varepsilon_{r} / \partial \rho^{2}\right)_{T}}{\left(\partial \varepsilon_{r} / \partial \rho\right)_{T}}-\frac{E^{2}}{\rho}\right]_{T} .
$$

On finding $\left(\partial \varepsilon_{r} / \partial \rho\right)_{T}$ and $\left(\partial^{2} \varepsilon_{r} / \partial \rho^{2}\right)_{T}$, we are in a position to calculate all the quantities occurring in (6). From (9) we get

$$
\left(\frac{\partial \varepsilon_{r}}{\partial \rho}\right)_{T}=\frac{3 A}{(1-A \rho)^{2}}
$$

and

$$
\left(\frac{\partial^{2} \varepsilon_{r}}{\partial \rho^{2}}\right)_{T}=\frac{6 A^{2}}{(1-A \rho)^{3}}
$$

IIence, by (12), the derivative of the squared electric ficld strength with respect to the density is

$$
\left(\frac{\partial E^{2}}{\partial \rho}\right)_{T}=\frac{2(1-A \rho)^{2}}{3 A \rho^{2} \varepsilon_{0} \beta_{\text {is }}}-E^{2} \frac{1+A \rho}{\rho(1-A \rho)} .
$$

We thus have all the elements required to calculate the square of the ultrasonic propagation velocity in the presence of the external electric field:

$$
\begin{gathered}
c_{E}^{2}=c_{0}^{2}-\frac{\kappa \varepsilon_{0}}{2}\left\{E^{2} \frac{\left(\varepsilon_{r}+2\right)\left(3 \varepsilon_{r}+2\right)}{9} \frac{3 A}{(1-A \rho)^{2}}\right. \\
\left.+\frac{\left(\varepsilon_{r}+2\right)^{2} \varepsilon_{r}}{9}\left[\frac{2(1-A \rho)^{2}}{3 A \rho^{2} \varepsilon_{0} \beta_{\mathrm{is}}}-E^{2} \frac{1+A \rho}{\rho(1-A \rho)}\right]\right\} .
\end{gathered}
$$

For the isothermal case, Eq. (16) finally leads to

$$
\begin{array}{r}
c_{E}^{2}=c_{0}^{2}-c_{0}^{2} \frac{\varepsilon_{r}\left(\varepsilon_{r}+2\right)^{2}(1-A \rho)^{2}}{27 A \rho} \\
-E^{2} \frac{\kappa \varepsilon_{0}}{18}\left[\frac{3 A\left(3 \varepsilon_{r}+2\right)\left(\varepsilon_{r}+2\right)}{(1-A \rho)^{2}}-\frac{\varepsilon_{r}(1+A \rho)\left(\varepsilon_{r}+2\right)^{2}}{\rho(1-A \rho)}\right] .
\end{array}
$$

Similarly, the squared velocily $c_{E}^{2}$ can be calculated for liquids with a non-zero permanent dipole moment $\boldsymbol{\mu}$. 
The dielectric constant as a function of temperature, volume and molar mass [1] is not explicitly dependent on the external field strength, but the field affects it indirectly by changes in strength on the magnitude of $\varepsilon_{r}$ in a given space point. The constant $\varepsilon_{r}$ is moreover frequency-dependent. For low frequencies $\varepsilon_{r}=\varepsilon_{0}$, whereas for high frequencies it is less, amounting to $\varepsilon_{r}(\omega)=\varepsilon_{\infty}$. Applying the expression for the local Onsager field one gets $c_{E}^{2}$ in a form valid for dipolar liquids.

Since in this case the local field strength is [4]:

$$
\boldsymbol{F}=\boldsymbol{E} \frac{2 \varepsilon_{r}^{2}+5 \varepsilon_{r}+2}{6 \varepsilon_{r}+3}
$$

$\partial\left(\varepsilon_{r} F^{2}\right) / \partial \rho$ can be written in the following form:

$$
\begin{aligned}
& \frac{\partial\left(\varepsilon_{r} F^{2}\right)}{\partial \rho}=E^{2}\left(\frac{\partial \varepsilon_{r}}{\partial \rho}\right)_{T} \frac{\left(2 \varepsilon_{r}^{2}+5 \varepsilon_{r}+2\right)^{2}}{9\left(2 \varepsilon_{r}+1\right)^{2}}+\left(\frac{\partial E^{2}}{\partial \rho}\right)_{T} \frac{\varepsilon_{r}\left(2 \varepsilon_{r}^{2}+5 \varepsilon_{r}+2\right)^{2}}{9\left(2 \varepsilon_{r}+1\right)^{2}} \\
&+E^{2}\left(\frac{\partial \varepsilon_{r}}{\partial \rho}\right)_{T} \frac{2 \varepsilon_{r}\left(2 \varepsilon_{r}^{2}+5 \varepsilon_{r}+2\right)\left(4 \varepsilon_{r}^{2}+4 \varepsilon_{r}+1\right)}{\left(2 \varepsilon_{r}+1\right)^{3}}
\end{aligned}
$$

On the basis of the Onsager's model [4], in which molecules are assumed to be spherical and short-range interactions are neglected, we arrive at the following relation between $\varepsilon_{r}$ and $\varepsilon_{\infty}$

$$
\varepsilon_{r}=\varepsilon_{\infty}+\frac{N_{A} \mu_{0}^{2} \rho}{9 \varepsilon_{0} M k T} \frac{\varepsilon_{r}\left(\varepsilon_{\infty}+2\right)^{2}}{2 \varepsilon_{r}+\varepsilon_{\infty}} .
$$

The values of $\left(\partial \varepsilon_{r} / \partial \rho\right)_{T}$ as well as $\left(\partial^{2} \varepsilon_{r} / \partial \rho^{2}\right)_{T}$ are readily calculated from Eq. (20), though the derivative $\left(\partial \rho / \partial \varepsilon_{r}\right)_{T}$ is more conveniently calculated with

$$
\rho=\frac{9 \varepsilon_{0} M k T}{\mu_{0}^{2} N_{A}} \frac{\left(2 \varepsilon_{r}^{2}-\varepsilon_{r} \varepsilon_{0}-\varepsilon_{\infty}^{2}\right)}{\varepsilon_{r}\left(\varepsilon_{\infty}+2\right)^{2}}
$$

We furthermore have

$$
\left(\frac{\partial \rho}{\partial \varepsilon_{r}}\right)_{T}=\frac{9 \varepsilon_{0} M k T}{\mu_{0}^{2} N_{A}} \frac{2 \varepsilon_{r}^{2}+\varepsilon_{\infty}^{2}}{\varepsilon_{r}^{2}\left(\varepsilon_{\infty}+2\right)^{2}}
$$

i.e.

$$
\left(\frac{\partial \varepsilon_{r}}{\partial \rho}\right)_{T}=\frac{N_{A} \mu_{0}^{2}}{9 \varepsilon_{0} M k T} \frac{\varepsilon_{r}^{2}\left(\varepsilon_{\infty}+2\right)^{2}-}{2 \varepsilon_{r}^{2}+\varepsilon_{\infty}^{2}}=\frac{\varepsilon_{r}\left(2 \varepsilon_{r}^{2}-\varepsilon_{r} \varepsilon_{\infty}-\varepsilon_{\infty}^{2}\right)}{\rho\left(2 \varepsilon_{r}^{2}+\varepsilon_{\infty}^{2}\right)},
$$

where use has been made of $(20)$. The other derivative is

$$
\begin{aligned}
\left(\frac{\partial^{2} \varepsilon_{r}}{\partial \rho^{2}}\right)_{T}= & \left(\frac{N_{A} \mu_{0}^{2}}{9 \varepsilon_{0} M k T}\right)^{2} \frac{2 \varepsilon_{r}^{3} \varepsilon_{\infty}^{2}\left(\varepsilon_{\infty}+2\right)^{4}}{\left(2 \varepsilon_{r}^{2}+\varepsilon_{\infty}^{2}\right)^{3}} \\
& =\frac{2 \varepsilon_{r} \varepsilon_{\infty}^{2}\left(2 \varepsilon_{r}^{2}-\varepsilon_{r} \varepsilon_{\infty}-\varepsilon_{\infty}^{2}\right)^{2}}{\rho^{2}\left(2 \varepsilon_{r}^{2}+\varepsilon_{\infty}^{2}\right)^{3}}
\end{aligned}
$$


Also, the two derivatives can be inserted into the formula (12) leading $t$ is the following expression for the magnitude of $\left(\partial E^{2} / \partial \rho\right)_{T}$

$$
\begin{gathered}
\left(\frac{\partial E^{2}}{\partial \rho}\right)_{T}=\frac{2\left(2 \varepsilon_{r}^{2}+\varepsilon_{\infty}^{2}\right)}{\rho \beta_{\mathrm{is}} \varepsilon_{0} \varepsilon_{r}\left(2 \varepsilon_{r}^{2}-\varepsilon_{r} \varepsilon_{\infty}-\varepsilon_{\infty}^{2}\right)} . \\
-\frac{E^{2}}{\rho} \cdot\left[1+\frac{2 \varepsilon_{\infty}^{2}\left(2 \varepsilon_{r}^{2}-\varepsilon_{r} \varepsilon_{\infty}-\varepsilon_{\infty}\right)^{2}}{\left(2 \varepsilon_{r}^{2}-\varepsilon_{\infty}^{2}\right)^{2}}\right] .
\end{gathered}
$$

With all the above derivatives we finally arrive at the following, general expression for the squared ultrasonic propagation velocity in dipolar dielectrics in the presence of an externally applied electric field:

$$
\begin{array}{r}
c_{E}^{2}=c_{0}^{2}-\frac{c_{0}^{2}}{9} \frac{\left(\varepsilon_{r}+2\right)^{2}\left(2 \varepsilon_{r}^{2}+\varepsilon_{\infty}^{2}\right)}{\left(2 \varepsilon_{r}^{2}-\varepsilon_{r} \varepsilon_{\infty}-\varepsilon_{\infty}^{2}\right)}-E^{2} \frac{\kappa \varepsilon_{0} \varepsilon_{r}\left(\varepsilon_{r}+2\right)}{18 \rho} \\
\times\left\{\frac{\left(3 \varepsilon_{r}+2\right)\left(2 \varepsilon_{r}^{2}-\varepsilon_{r} \varepsilon_{\infty}-\varepsilon_{\infty}^{2}\right)}{\left(\varepsilon_{r}^{2}+\varepsilon_{\infty}^{2}\right)}-\left(\varepsilon_{r}+2\right)\left[1+\frac{2 \varepsilon_{\infty}^{2}\left(2 \varepsilon_{r}^{2}-\varepsilon_{r} \varepsilon_{\infty}-\varepsilon_{\infty}^{2}\right)}{\left(2 \varepsilon_{r}^{2}+\varepsilon_{\infty}^{2}\right)^{2}}\right]\right\} .
\end{array}
$$

\section{The phase method of measurement of the ultrasonic propagation velocity and its variations in liquid dielectrics}

Theoretically $[6-8]$, the changes in ultrasonic propagation velocity due to the influence of an external DC electric field in a liquid dielectr $c$ are predicted to be very small. Accordingly, in our measurements, we had recisurse to the phase method - one of the most highly sensitive methods available $[9,10]$. Using a high quality digital phase detector and applying computer technique for data processing, we achieved a very high degree of accuracy in our measurements.

A block scheme of the method is given in Fig. 1. The measuring vessel contained two branches (ultrasonic paths), one of which was the reference path and the other served as measuring path. Both paths were filled with the same liquid dielectric in which two mutually independent bearrs of continuous ultrasonic aves from emitter transducers $(1,2)$ attached to a stabilized frequency generator were made to propagate. The lengths of the two paths were equal, amounting $t o l=$ $0.248 \mathrm{~m}$.

The measuring vessel was in contact with a system stabilizing the temperature to within $0.05 \mathrm{~K}$. The receiver transducers $(3,4)$ were att:ched to the illputs of a digital phase detector, the output voltage of which was proportional to the difference in phases between the two input signals, with phases $\phi_{0}$ (in the reference branch) and $\phi_{1}$ (in the measuring branch).

The determination of the phase velocity $c_{0}$ (where the subscript " 0 ". denotes the value of $c$ at zero external field), with regard to the relation

$$
c_{0}=\frac{l f}{n_{0}}
$$




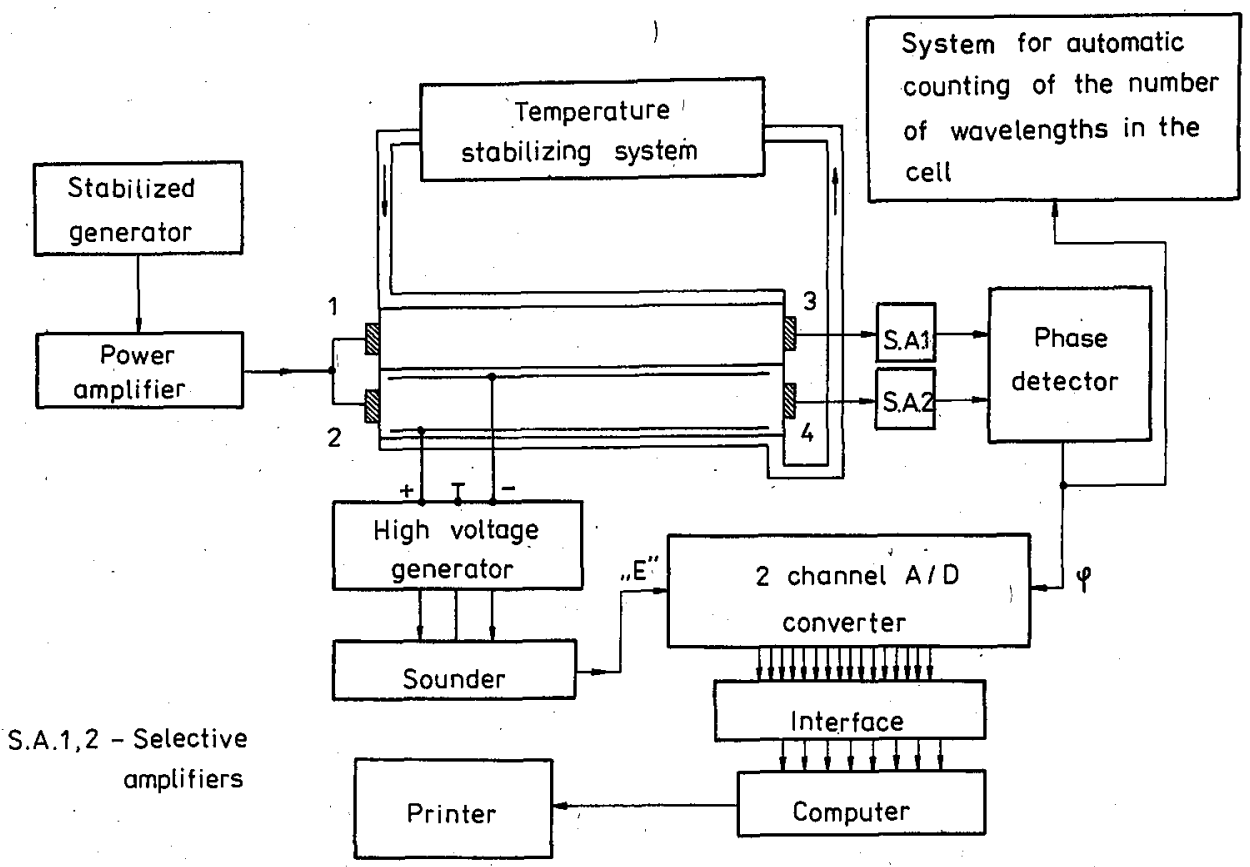

Fig. 1. Block diagram of the setup used in measuring the phase velocity and its variations under the influence of an external electric field. Measurements performed by the phase method.

involves measurements of

1) the patil $l$ between the transducers,

2) the frequency of the ultrasonic wave, and

3) the number of wavelengths $n$ in the vessel (not necessarily an integer).

The digital phase detector used by us - by way of the changes in vollage at its output - permitted the determination of the change in phase or rather the difference in phase between the two signals coming, respectively, from the reference path and from the measuring path, where the change is caused by action of the external field on the liquid.

As long as reflection of the sine wave between the transducers, is insignificant [11] the absolute variations in propagation velocity amounts to

$$
\Delta c=\frac{-\Delta \phi l f}{2 \pi n_{0}^{2}}
$$

Thus, all these quantities $\left(\Delta \phi, l, f\right.$, and $\left.n_{0}\right)$ affect the accuracy achieved in determining the change in the velocity of the wave. When applying the phase method, 
use can be made of the formula

$$
\frac{\Delta c}{c_{0}}=\frac{-\Delta \phi}{2 \pi n_{0}}=\frac{-\Delta \phi}{\phi_{0}}
$$

It shows that the accuracy achieved in measuring the relative change in propagation velocity of the wave is dependent on that achieved in measuring the total phase $\phi_{0}$ and the change in phase $\Delta \phi$ in the vessel only. Thus the length $l$ of the latter and the frequency $f$ do not affect the magnitude of $\Delta c / c_{0}$ directly. However, the longer the vessel and the higher the frequency (the transducers can operate on an odd harmonic) the greater is the phase $\phi_{0}$ thus enhancing the sensitivity of the measuring setup at a given sensitivity of the phase detector.

When taking readings of the output voltage from the phase detector with a computer by way of an 8-bit analog-digital transducer, the smallest change in phase accessible to observation is

$$
\Delta \phi_{\min }=\frac{2 \times 2 \pi}{9 \times 128}\left[\frac{\mathrm{rd}}{\mathrm{bit}}\right]=10.9 \times 10^{-3}\left[\frac{\mathrm{rd}}{\mathrm{bit}}\right] .
$$

This means that - in the case of carbon tetrachloride, and $f=1.6 \mathrm{MIIz}$, ' $l=0.248 \mathrm{~m}, T=293 \mathrm{~K}$ and $c_{0}=938 \mathrm{~m} / \mathrm{s}$ - the smallest observable change in velocity would be

$$
\left|\Delta c_{\min }\right|=\left|\frac{\Delta \phi_{\min } c_{0}}{2 \pi n_{0}+\Delta \phi_{\min }}\right| \approx \frac{10.9 \times 10^{-3} \times 938}{2 \pi \times 423}=3.8 \times 10^{-3}[\mathrm{~m} / \mathrm{s}],
$$

corresponding to a measurement of the relative change in phase velocity $\Delta c / c$ with an accuracy of $4.05 \times 10^{-6}$.

It should be noted that the accuracy of the measuring device can be much higher but the accuracy in measuring the velocity is limited by difficulties in maintaining the physical properties of the liquid constant (its temperature, density, homogeneity, flowlessness, etc.) and by inhomogeneities of the acoustic field.

\section{The experimental results and their analysis}

Our experimental study of the effect of an externally applied DC electric field on the propagation velocity of ultrasonic waves was carried out on mineral transformer oil, carefully purified with aluminum trioxide, dried on metallic sodium, and additionally "electric field-cleaned" [12].

Measurements were performed for electric field strengths in the range from 0 to $308 \mathrm{kV} / \mathrm{m}$ and temperatures from 253 to $293 \mathrm{~K}$. The ultrasonic frequency amounted to $1.6 \mathrm{MIIz}$. The absolute value of the velocity was determined with $\mathrm{Eq}$. (27) and its variation with Eq. (28).

Figures (2-4) exemplify time-dependent recordings of the changes in propagation velocity in the electric field for different field strengths.

The duration of each measurement amounted to about 2 minutes. The graphs (Figs. 2-4) show that after a lapse of time, which depends on the magnitudes of the experimental parameters, the ultrasonic velocity settles at a well defined level. 


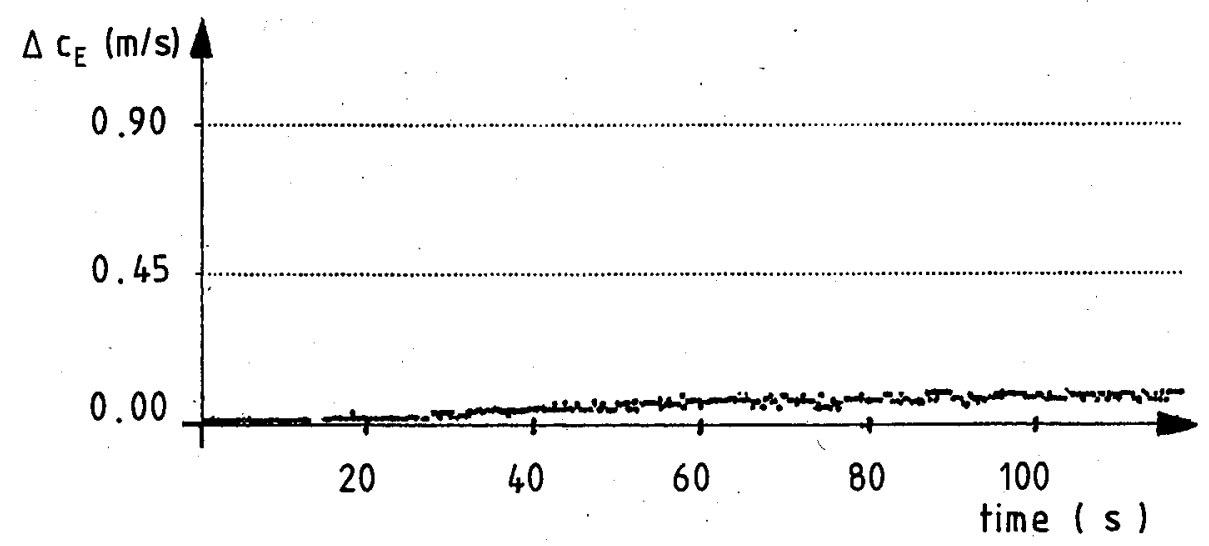

Fig. 2. Time-variations in ultrasonic propagation velocity for transformer oil: $n_{0}=$ 248.24 - the number of wavelengths in the measuring vessel; $c_{0}=1598.45 \mathrm{~m} / \mathrm{s}$; $E=77 \mathrm{kV} / \mathrm{m} ; T=253 \mathrm{~K}$.

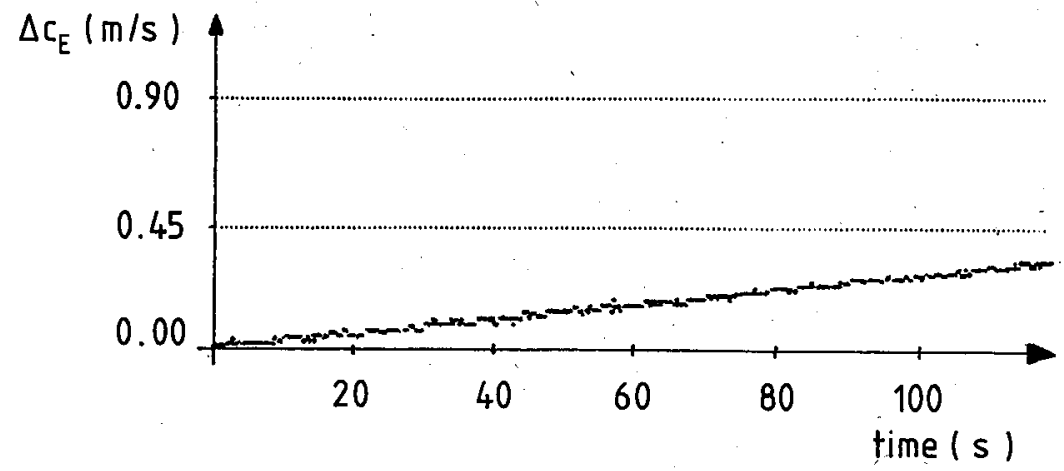

Fig. 3. Time-variations in ultrasonic propagation velocity, for transformer oil: $n_{0}=$ $248.24 ; \quad c_{0}=1598.45 \mathrm{~m} / \mathrm{s} ; \quad E=154 \mathrm{kV} / \mathrm{m} ; \quad T=253 \mathrm{~K}$. 


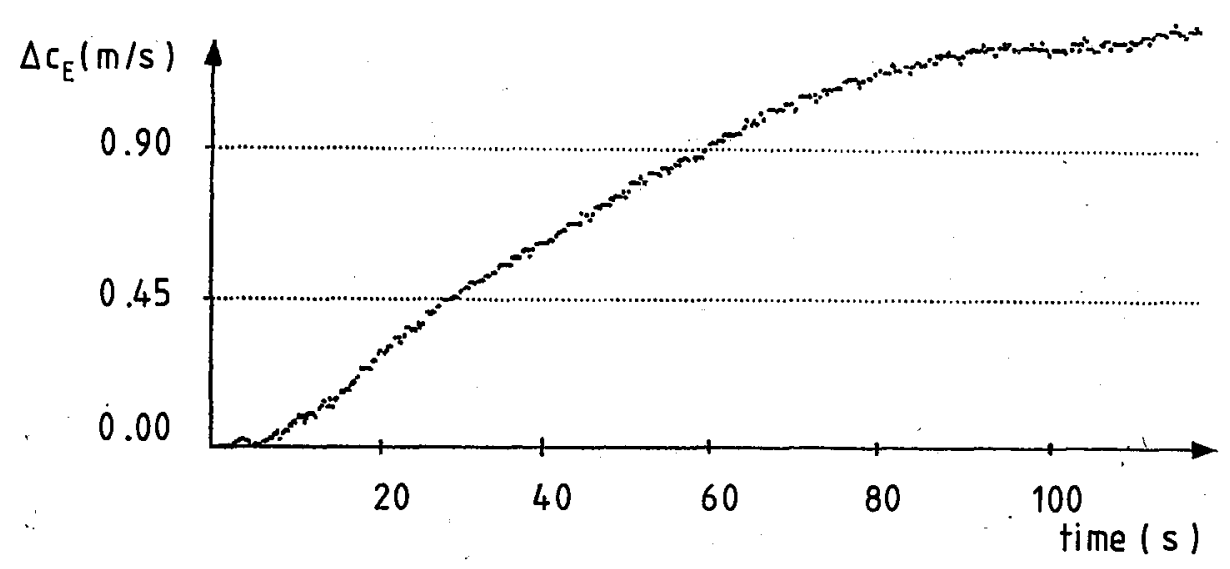

Fig. 4. Time-variations in ultrasonic propagation velocity, for transformer oil: $n_{0}=$ $248.24 ; \quad c_{0}=1598.45 \mathrm{~m} / \mathrm{s} ; \quad E=308 \mathrm{kV} / \mathrm{m} ; \quad T=253 \mathrm{~K}$.

The only information we had recourse to in our further analysis concerned the magnitude of $\Delta c_{E}$ in the steady state. We assumed for each measurement a conventional time equal to 117 seconds. On the basis of some tens of $\Delta c_{E}$-recordings, taken in different physical conditions of the field strength $E$ and temperature $T$ we plotted $\Delta c_{E}$ versus $E$ for the transformer oil dealing with $T$ as a parameter (see Fig. 5). Fig. 6 shows an example of $\Delta c_{E}=f(T)$, with $E$ as a parameter.

In plotting the graph of Fig. 6 we applied Lagrange interpolation $[13,14]$ the principle of which is that the graphs resulting from this mathematical operation shall traverse the experimental points.

The formulae for the squared propagation velocity derived by us from the change in free energy on application of the external DC electric ficld, in conjunction with the present experimental results, enable us to announce several gencral conclusions.

The absolute change in propagation velocity in the viscous medium, in various physical conditions, is of the order of $\Delta c_{E} \approx\left(10^{-2} \div 1\right) \mathrm{m} / \mathrm{s}$; hence, its relative variation amounts to $\Delta c / c_{0} \approx\left(10^{-5} \div 10^{-4}\right)$. On the other hand, the relative variations evaluated theoretically from the Eqs. (17 and 26) are of the order of $\approx 10^{-7}$ for typical non-dipolar and dipolar diclectrics in the presence of a DC elcctric field.

Thus, the divergence between the experimental results and the theoretical evaluations is considerable, suggesting the existence (beside the ficld-induced change in free energy) of yet other factors modifying the propagation velocity in the liquid. The measurements, however, reveal two distinct trends in the behaviour of the velocity variations: for a given temperature the effect is the greater the stronger is the field applied to the dielectric. This trend was observed for all the temperatures; however, the variation $\Delta c_{E}$ was maximal for the lowest temperature $T=253 \mathrm{~K}$ but decreased with growing $T$. 


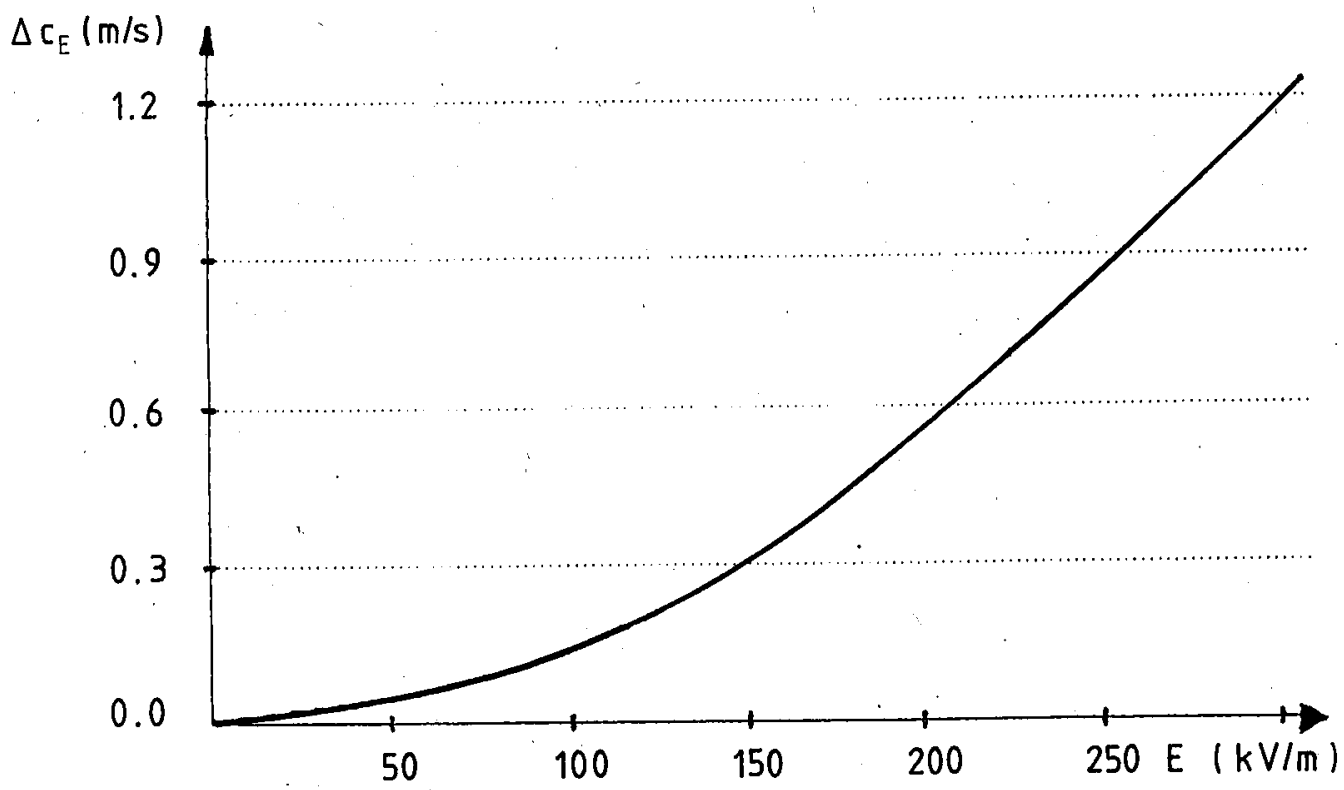

Fig. 5. $\Delta c_{E}$ vs. $E$ in degassed transformer oil for $T=253 \mathrm{~K}$.

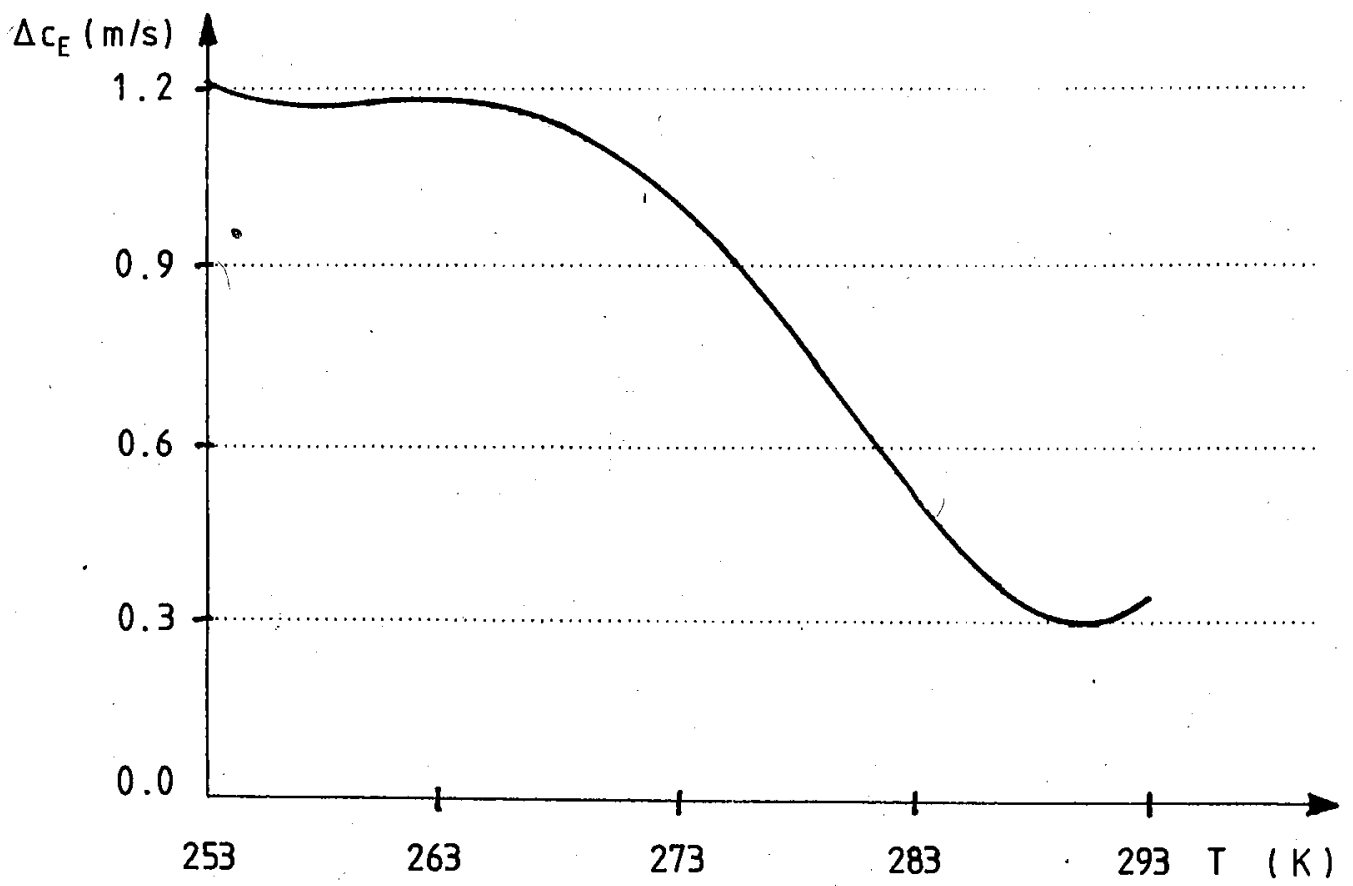

Fig. 6. $\Delta c_{E}$ vs. $T$ in degassed transformer oil, for $E=308 \mathrm{kV} / \mathrm{m}$. 
The thcory of dielectrics shows that variations in free energy related with dipole polarization usually occur on a microsecond time-scale; whereas our measurcments prove that the chariges in propagation velocity in the oil occurred on a time-scalc of as much as several tens of seconds, suggesting that the electric field acts on the velocity by affecting the free energy and, moreover, by way of yet other mochanisms.

Its elucidation will be the object of our other work, both theoretical and expcrimental.

Also, the theoretical formulae show that for electric fields in the range $(0 \div$ $\left.E_{\max }\right)$ the magnitude of $\left(\partial E^{2} / \partial \rho\right)_{T}$ is practically independent of the field strength, i.e. that the change in density due to electrostriction is proportional to $E^{2}$.

\section{Reforences}

[1] I. Werle, Termodynamika Senomenologiczna, PWN, Warszawa 1957 (in Polish).

[2] W. Van Dael, in: Pure and Applied Chemistry, Chap. 11, eds. B. Leneindre, B. Vodar, Reprinted from Experimental Thermodynamics, vol. 2. 1975.

[3] I.G. Mikhaylov, V.A. Solovyov, Yu.P. Syrnikov, Osnovy molekularnoy akustiki, Izd. Nauka, Moskva 1964 (in Russian).

[4] A. Chelkowski, Fizyka dielektryków, PWN, Warszawa 1979. (in Polish).

[5] I.E. Tamm, Podslawy leorii eleklryczności, WNT, Warszawa 1967 (in Polish).

[6] A. Skumicl, M. Labowski, Archiwum Akuslyki, 22, 1 (1987) (in Polish).

[7] W. Schaaffs, Molckularakuslik, Springer-Verlag, Berlin, Göttingen, Heidelberg 1963.

[8] I. Malecki, Teoria fal i uktadów akuslycznych, PWN, Warszawa 1964 (in Polish).

[9] E.P. Papadakis, in Ullrasonic Velocity and Altenuation: Measurement Melhods with Scienlific and Industrial Applications, Vol. 9. eds. W.P. Mason, R.N. Thurston, Academic Press, New York 1975, p. 277.

[10] A.Śliwiński, Poslepy Fizyki Molekularnej, 1987. (in Polish).

[11] J. Wchr, Pomiary pręllości i tlumienia fal ultradźwiękowych, PWN, Warszawa 1972 (in Polish).

[12] I. Adamczewski, Jonizacja i przewodniclwo cieklych dieleklryków, PWN, Warszawa 1965 (in Polish).

[13] J. i M. Jankowscy, Przegląd melod i algorylmów numerycznych, WNT, Warszawa 1981 (in Polish).

[14] B.P. Demidowicz, F.A. Maron, Melody numeryczne, PWN, Warszawa 1965 (in Polish). 